\title{
Smarandachely Adjacent-Vertex-Distinguishing Proper Edge Chromatic Number of $C_{m} \vee K_{n}$
}

\author{
Shunqin Liu \\ School of Information \& Technology, Xiamen University Tan Kah Kee College, Zhangzhou, China
}

Email address:

pytxlx@163.com

To cite this article:

Shunqin Liu. Smarandachely Adjacent-Vertex-Distinguishing Proper Total Coloring Number of $C_{m} \vee K_{n}$. Applied and Computational Mathematics. Vol. 5, No. 5, 2016, pp. 202-206. doi: 10.11648/j.acm.20160505.13

Received: September 23, 2016; Accepted: October 13, 2016; Published: October 17, 2016

\begin{abstract}
According to different conditions, researchers have defined a great deal of coloring problems and the corresponding chromatic numbers. Such as, adjacent-vertex-distinguishing total chromatic number, adjacent-vertex-distinguishing proper edge chromatic number, smarandachely-adjacent-vertex-distinguishing proper edge chromatic number, smarandachely-adjacent-vertex-distinguishing proper total chromatic number. And we focus on the smarandachely adjacent-vertex-distinguishing proper edge chromatic number in this paper, study the smarandachely adjacent-vertex-distinguishing proper edge chromatic number of joint graph $C_{m} \vee K_{n}$.
\end{abstract}

Keywords: Graph Theory, Joint Graph, Smarandachely Adjacent-Vertex-Distinguishing Proper Edge Chromatic Number

\section{Introduction}

Coloring problem in graph theory, is one of the most famous NP-complete problems. Four color conjecture which is one of the world's three major mathematical conjecture says that each map can be used only four colors to dye, and no two adjacent areas dyed the same color. In the spring of 1976, with the help of the computer, the four color conjecture was proved. The conjecture finally became a theorem. The significance of graph coloring theory is much more than that. Known to all, coloring problems can solve many problems such as scheduling problem, time tabling, transportation, arrangement, circuit design and storage problems.

In recent years, more and more coloring problems was put forward by experts of graph theory, such as proper-adjacentvertex-distinguishing edge coloring, proper -adjacentvertex-distinguishing total coloring, smarandachely adjacentvertex-distinguishing proper edge coloring.

\section{Smarandachely}

\section{Adjacent-Vertex-Distinguishing Proper Edge Coloring}

Definition 1[1] A k-proper edge coloring of a graph $G$ is a mapping $f$ from $E(G)$ to $\{1,2, \ldots, k\}$ that satisfies the condition described as below:

For $\forall e_{i}, e_{j} \in E(G), e_{i} \neq e_{j}$, if $e_{i}, e_{j}$ have a common end vertex, then $f\left(e_{i}\right) \neq f\left(e_{j}\right)$.

The number $\min \{k \mid G$ has a k-proper edge coloring of graph $G\}$ is called the proper edge chromatic number of $G$, denoted by $\chi(G)$. If $f\left(e_{i}\right)=l$, then we call the number $l$ to be the color of edge $e_{i}$

Definition 2[1] A k-proper edge coloring $f$ is called a k-proper-adjacent-vertex-distinguishing proper edge coloring, short for k-AVDPEC when $f$ satisfies condition described as below:

Denote $C(u)=\{f(u v) \mid v \in V(G) \wedge u v \in E(G)\}$ for every vertex $u \in V(G)$, if for $\forall u, v \in V(G), u v \in E(G)$, we have $C(u) \neq C(v)$.

The number $\min \{k \mid G$ has a k-proper-adjacentvertex-distinguishing proper edge coloring $\}$ is called the adjacent -vertex-distinguishing proper edge chromatic number and denoted by $\chi_{a}^{\prime}(G)$. Set $C(u)$ is called the color set of the vertex.

Definition 3[1] A k-proper edge coloring $f$ is called a smarandachely adjacent- vertex-distinguishing proper edge 
coloring, short for k-SA when $f$ satisfies conditions described as below:

Denote $C(u)=\{f(u v) \mid v \in V(G) \wedge u v \in E(G)\}$ for every vertex $u \in V(G)$, if $\forall u, v \in V(G), u v \in E(G)$, we have $C(u) \not \subset C(v)$ and $C(v) \not \subset C(u)$ mean well.

The number $\min \{k \mid G$ has a $k-S A\}$ is called the smarandachely adjacent-vertex-distinguishing proper edge chromatic number of $G$, denoted by $\chi_{s a}^{\prime}(G)$.

It's obviously of below conclusion: when $G$ have a vertex who's degree equal to 1 , then $G$ have no k-SA for all nature number $\mathrm{k}$.

The paper use $C_{m}$ to denote the graph of circle with $m$ vertices and $K_{n}$ to denote the complete graph with $n_{\text {vertices, }}$ use $C_{m} \vee K_{n}$ to denote the joint graph of $C_{m}$ and $K_{n}$. We denote the vertex sets and the edge set of the graphs such that:

$V\left(C_{m}\right)=\left\{u_{1}, u_{2}, \cdots, u_{m}\right\} \quad, \quad E\left(C_{m}\right)=\left\{\left(u_{i-1} u_{i}\right) \mid 2 \leq i \leq m\right\} \cup\left\{\left(u_{m} u_{1}\right)\right\}$, $V\left(K_{n}\right)=\left\{v_{1}, v_{2}, \cdots, v_{n}\right\}$.

$d(u)$ is the degree of the vertex $u, \Delta$ is the maximum degree of the graph discussed, $\mathrm{S}$ is the universal set of the color used, that $S=\underset{u \in V(G)}{\cup} C(u), \bar{C}(u)$ is the complement of $C(u)$.

Lemma 1 [1] If $G$ denote the graph have no one degree vertex, then

1) $\chi_{s a}^{\prime}(G) \geq \chi_{a}^{\prime}(G)$, if $G$ is a regular graph then $\chi_{s a}^{\prime}(G)=\chi_{a}^{\prime}(G)$.

2) $\chi_{s a}^{\prime}(G) \geq \Delta+1$.

Lemma 2 [1] If $K_{n}$ is the complete graph with vertices $n$, $n \geq 3$, then

$$
\chi_{a}^{\prime}\left(K_{n}\right)=\left\{\begin{array}{cccc}
n, & n & \text { is } & \text { odd } \\
n+1, & n & \text { is } & \text { even }
\end{array} .\right.
$$

Lemma 3[2] If $K_{n}$ is the complete graph with vertices $n$, $n \geq 4$ and $n$ is even, then $\chi\left(K_{n}\right)=n-1$.

\section{Smarandachely \\ Adjacent-Vertex-Distinguishing Proper Edge Coloring of $\boldsymbol{C}_{\boldsymbol{m}} \vee \boldsymbol{K}_{\boldsymbol{n}}$}

Theorem 1 If $m \geq 3, n \geq 3, m, n$ are both even, then $C_{m} \vee K_{n}$ have no $m+n-S A$.

Proof Suppose that $C_{m} \vee K_{n}$ have a $m+n-S A$, then $S=\{1,2, \cdots, m+n\}$. Be aware of the facts that: $d\left(v_{1}\right)=d\left(v_{2}\right)=\cdots=d\left(v_{n}\right)=m+n-1$

Then $\left|C\left(v_{1}\right)\right|=\left|C\left(v_{2}\right)\right|=\cdots=\left|C\left(v_{n}\right)\right|=m+n-1$

$$
\left|\bar{C}\left(v_{1}\right)\right|=\left|\bar{C}\left(v_{2}\right)\right|=\cdots=\left|\bar{C}\left(v_{n}\right)\right|=1
$$

$\forall i \neq j, \quad 1 \leq i \leq n, \quad 1 \leq j \leq n, \quad v_{i}$ is adjacent to $v_{j}$, so $C\left(v_{i}\right) \neq C\left(v_{j}\right)$, so

$$
\forall i \neq j \quad 1 \leq i \leq n, \quad 1 \leq j \leq n, \bar{C}\left(v_{i}\right) \neq \bar{C}\left(v_{j}\right)
$$

Inferred from (1) and (2), then each vertex $v_{i}(1 \leq i \leq n)$ has one different color from each other.

Then we may as well suppose that $i \notin \bar{C}\left(v_{i}\right)$, for $\forall 1 \leq i \leq n$

Then it must have the result such as $\{1,2, \cdots, n\} \subset C\left(u_{j}\right)$, $\forall 1 \leq j \leq m$ (otherwise, if there exists a color $k, 1 \leq k \leq n$, and exists a vertex $u_{j}$ satisfies $k \notin C\left(u_{j}\right)$, then we can deduce the result such as $C\left(u_{j}\right) \subset C\left(v_{i}\right), \forall 1 \leq i \leq n$, but the vertex $u_{j}$ is adjacent to the vertex $v_{i}$, this result is in contradiction with the definition of $k-S A$ ). Now we consider all vertices of the whole graph $C_{m} \vee K_{n}$ who satisfy $f(u)=1$, according to previous discussion, except vertex $v_{1}$, all the remaining $m+n-1$ vertices of the graph $C_{m} \vee K_{n}$ satisfied the condition $f(u)=1$, that is to say, except the vertex $v_{1}$, the remaining $m+n-1$ vertices form a matching, this result is in contradiction with the fact such as that $m+n-1$ is odd.

So, $C_{m} \vee K_{n}$ have no $m+n-S A$.

Theorem 2 If $n \geq 6, n$ is even, then

$$
\chi_{s a}^{\prime}\left(C_{n-2} \vee K_{n}\right)=2 n-1 .
$$

Proof Be aware of the fact that the maximum degree of graph $C_{n-2} \vee K_{n}$ is $\Delta=2 n-3$, by the 2) of Lemma 1, we get the result such as $\chi_{s a}^{\prime}\left(C_{n-2} \vee K_{n}\right) \geq 2 n-2$, also because of the fact that $n$ is even, then by theorem 1 , the graph $C_{n-2} \vee K_{n}$ have no $(2 n-2)-S A$, so we get the result such as $\chi_{s a}^{\prime}\left(C_{n-2} \vee K_{n}\right) \geq 2 n-1$.

For the subgraph $K_{n}$, by lemma 3 , there is a $n-1$-proper edge coloring on $K_{n}$, that is to say, there is a mapping $g$ from $E\left(K_{n}\right)$ to $\{1,2, \ldots, n-1\}$ satisfied that $\forall e_{i}, e_{j} \in E\left(K_{n}\right)$, $e_{i} \neq e_{j}$, if $e_{i}, e_{j}$ have a common end vertex, then $g\left(e_{i}\right) \neq g\left(e_{j}\right)$.

Now we define a mapping $f$ from $E\left(C_{n-2} \vee K_{n}\right)$ to $\{1,2, \ldots, n-1, n, n+1, n+2, \cdots, 2 n-1\}$ described as below:

If $e \in K_{n}$, then $f(e)=g(e)$, then $\{1,2, \ldots, n-1\}$ $\subset C\left(v_{i}\right)$, for $\forall 1 \leq i \leq n$,

$$
\begin{aligned}
& f\left(u_{1} v_{j}\right)=n+j-1, \quad 1 \leq j \leq n, \\
& f\left(u_{2} v_{j}\right)=n+j, \quad 1 \leq j \leq n-1, \quad f\left(u_{2} v_{n}\right)=n, \\
& f\left(u_{3} v_{j}\right)=n+j+1, \quad 1 \leq j \leq n-2,
\end{aligned}
$$




$$
\begin{gathered}
f\left(u_{3} v_{n-1}\right)=n, \quad f\left(u_{3} v_{n}\right)=n+1, \\
f\left(u_{4} v_{j}\right)=n+j+2, \quad 1 \leq j \leq n-3, \\
f\left(u_{4} v_{n-2}\right)=n, \quad f\left(u_{4} v_{n-1}\right)=n+1, \\
f\left(u_{4} v_{n}\right)=n+2, \\
\quad \ldots \\
f\left(u_{n-2} v_{1}\right)=2 n-3, \quad f\left(u_{n-2} v_{2}\right)=2 n-2, \\
f\left(u_{n-2} v_{3}\right)=2 n-1, \\
f\left(u_{n-2} v_{j}\right)=n+j-4, \quad 4 \leq j \leq n, \\
f\left(u_{i-1} u_{i}\right)=i, \quad 2 \leq i \leq n-2, f\left(u_{n-2} u_{1}\right)=1 .
\end{gathered}
$$

By the definition of $f$, we get the $C(u)$ and $\bar{C}(u)$ of every vertex of the graph such as below:

$$
\begin{aligned}
& C\left(v_{1}\right)=\{1,2,3, \cdots, n-1\} \cup\{n, n+1, n+2, \cdots, 2 n-3\} \\
& \bar{C}\left(v_{1}\right)=\{2 n-2,2 n-1\}, \bar{C}\left(v_{2}\right)=\{n, 2 n-1\}, \\
& \bar{C}\left(v_{3}\right)=\{n, n+1\}, \bar{C}\left(v_{4}\right)=\{n+1, n+2\}, \\
& \cdots \\
& \bar{C}\left(v_{n}\right)=\{2 n-3,2 n-2\}, \\
& C\left(u_{1}\right)=\{n, n+1, n+2, \cdots, 2 n-1\} \cup\{1,2\} \\
& \bar{C}\left(u_{1}\right)=\{3,4, \cdots, n-1\}=\{1,2, \cdots, n-1\} \backslash\{1,2\}, \\
& \bar{C}\left(u_{2}\right)=\{1,2, \cdots, n-1\} \backslash\{2,3\}, \\
& \quad \cdots \\
& \bar{C}\left(u_{i}\right)=\{1,2,3, \cdots, n-1\} \backslash\{i, i+1\}, \forall 2 \leq i \leq n-3, \\
& \bar{C}\left(u_{n-2}\right)=\{1,2, \cdots, n-1\} \backslash\{n-2,1\} .
\end{aligned}
$$

We can see that

$$
\begin{aligned}
& \bar{C}\left(u_{i}\right) \not \subset \bar{C}\left(v_{j}\right), \quad 1 \leq i \leq n-2, \quad 1 \leq j \leq n, \\
& \bar{C}\left(v_{j}\right) \not \subset \bar{C}\left(u_{i}\right), \quad 1 \leq i \leq n-2, \quad 1 \leq j \leq n, \\
& \bar{C}\left(u_{i}\right) \not \subset \bar{C}\left(u_{j}\right), \quad 1 \leq i, j \leq n-2,|i-j|=1, \\
& \bar{C}\left(u_{1}\right) \not \subset \bar{C}\left(u_{n-2}\right), \quad \bar{C}\left(u_{n-2}\right) \not \subset \bar{C}\left(u_{1}\right), \\
& \bar{C}\left(v_{i}\right) \not \subset \bar{C}\left(v_{j}\right), \quad 1 \leq i \leq n, \quad 1 \leq j \leq n, \quad i \neq j .
\end{aligned}
$$

So the given $f$ is a $(2 n-1)-S A$ for $C_{n-2} \vee K_{n}$. That is to say

$$
\chi_{s a}^{\prime}\left(C_{n-2} \vee K_{n}\right)=2 n-1 .
$$

Example 1 For $n=6$, then $\chi_{s a}^{\prime}\left(C_{4} \vee K_{6}\right)=11$.

In fact, for the subgraph $K_{6}$ of $C_{4} \vee K_{6}$, we defined a 5-proper edge coloring $g$ such that:

$$
\begin{aligned}
& g\left(v_{1} v_{2}\right)=g\left(v_{3} v_{6}\right)=g\left(v_{4} v_{5}\right)=1, \\
& g\left(v_{1} v_{3}\right)=g\left(v_{2} v_{4}\right)=g\left(v_{5} v_{6}\right)=2, \\
& g\left(v_{1} v_{4}\right)=g\left(v_{3} v_{5}\right)=g\left(v_{2} v_{6}\right)=3, \\
& g\left(v_{1} v_{5}\right)=g\left(v_{4} v_{6}\right)=g\left(v_{2} v_{3}\right)=4, \\
& g\left(v_{1} v_{6}\right)=g\left(v_{2} v_{5}\right)=g\left(v_{3} v_{4}\right)=5 .
\end{aligned}
$$

Obviously, every adjacent edge have different colors, so $g$ is a 5-proper edge coloring of $K_{6}$.

Then, for graph $C_{4} \vee K_{6}$, we define the mapping $f$ from $E\left(C_{4} \vee K_{6}\right)$ to $\{1,2,, 3, \ldots, 10,11\}$ described as below:

For edge $v_{i} v_{j}, f\left(v_{i} v_{j}\right)=g\left(v_{i} v_{j}\right), \quad 1 \leq i \neq j \leq 6$,

$$
\begin{aligned}
& f\left(u_{1} v_{j}\right)=n+j-1, \quad 1 \leq j \leq 6, \\
& f\left(u_{2} v_{j}\right)=n+j, \quad 1 \leq j \leq 5, \quad f\left(u_{2} v_{6}\right)=6, \\
& f\left(u_{3} v_{j}\right)=n+j+1, \quad 1 \leq j \leq 4, \\
& f\left(u_{3} v_{5}\right)=6, \quad f\left(u_{3} v_{6}\right)=7, \\
& f\left(u_{4} v_{j}\right)=n+j+2, \quad 1 \leq j \leq 3, \\
& f\left(u_{4} v_{4}\right)=6, \quad f\left(u_{4} v_{5}\right)=7, f\left(u_{4} v_{6}\right)=8, \\
& f\left(u_{i-1} u_{i}\right)=i, \quad 2 \leq i \leq 4, f\left(u_{n-2} u_{1}\right)=1 .
\end{aligned}
$$

We can see that the $C(u)$ and $\bar{C}(u)$ of every vertex for graph $C_{4} \vee K_{6}$ are described as below:

$$
\begin{aligned}
& C\left(v_{1}\right)=\{1,2,3,4,5\} \cup\{6,7,8,9\}, \\
& \bar{C}\left(v_{1}\right)=\{10,11\}, \\
& \bar{C}\left(v_{2}\right)=\{6,11\}, \bar{C}\left(v_{3}\right)=\{6,7\}, \\
& \bar{C}\left(v_{4}\right)=\{7,8\}, \bar{C}\left(v_{5}\right)=\{8,9\}, \bar{C}\left(v_{6}\right)=\{9,10\}, \\
& \bar{C}\left(u_{1}\right)=\{3,4,5\}, \bar{C}\left(u_{2}\right)=\{1,4,5\}, \\
& \bar{C}\left(u_{3}\right)=\{1,2,5\}, \bar{C}\left(u_{4}\right)=\{2,3,5\} .
\end{aligned}
$$

We can see that the color set of the adjacent vertices meet the requirements of definition[3], so $f$ is a $11-S A$ for $C_{4} \vee K_{6}$, then $\chi_{s a}^{\prime}\left(C_{4} \vee K_{6}\right)=11$.

Theorem 3 If $n \geq 4, n$ is even, then 


$$
\chi_{s a}^{\prime}\left(C_{n-1} \vee K_{n}\right)=2 n-1 .
$$

Proof Because of the fact that the maximum degree of the graph $C_{n-1} \vee K_{n}$ is $\Delta=2 n-2$, by the 2) of Lemma 1, we get the result such as $\chi_{s a}^{\prime}\left(C_{n-1} \vee K_{n}\right) \geq 2 n-1$.

For the subgraph $K_{n}$ of the graph $C_{n-1} \vee K_{n}$, by lemma 3, there is a $n-1$-proper edge coloring on $K_{n}$, that is to say, there is a mapping $g$ from $E\left(K_{n}\right)$ to $\{1,2, \ldots, n-1\}$ that is satisfied the conditions such as:

$\forall e_{i}, e_{j} \in E\left(K_{n}\right), e_{i} \neq e_{j}$, if $e_{i}, e_{j}$ have a common end vertex, then $g\left(e_{i}\right) \neq g\left(e_{j}\right)$.

Now we define a mapping $f$ from $E\left(C_{n-1} \vee K_{n}\right)$ to $\{1,2, \ldots, n-1, n, n+1, n+2, \cdots, 2 n-1\}$ as below:

If $e \in K_{n}$, then $f(e)=g(e)$, then $\{1,2, \ldots, n-1\}$ $\subset C\left(v_{i}\right), \forall 1 \leq i \leq n$,

$$
\begin{aligned}
& f\left(u_{1} v_{j}\right)=n+j-1, \quad 1 \leq j \leq n, \\
& f\left(u_{2} v_{j}\right)=n+j, \quad 1 \leq j \leq n-1, \quad f\left(u_{2} v_{n}\right)=n, \\
& f\left(u_{3} v_{j}\right)=n+j+1, \quad 1 \leq j \leq n-2, \\
& f\left(u_{3} v_{n-1}\right)=n, \quad f\left(u_{3} v_{n}\right)=n+1, \\
& \ldots \\
& f\left(u_{n-1} v_{1}\right)=2 n-2, \quad f\left(u_{n-1} v_{2}\right)=2 n-1, \\
& f\left(u_{n-1} v_{j}\right)=n+j-3, \quad 3 \leq j \leq n, \\
& f\left(u_{i-1} u_{i}\right)=i, \quad 2 \leq i \leq n-1 \quad f\left(u_{n-1} u_{1}\right)=1, \quad 2 \leq i \leq n-1 .
\end{aligned}
$$

By the definition of $f$, we get that the $C(u)$ and $\bar{C}(u)$ of every vertex as below:

$$
\begin{aligned}
& C\left(v_{1}\right)=\{1,2, \cdots, n-1\} \cup\{n, n+1, \cdots, 2 n-2\}, \\
& \bar{C}\left(v_{1}\right)=\{2 n-1\}, \bar{C}\left(v_{2}\right)=\{n\}, \\
& \bar{C}\left(v_{j}\right)=\{n+j-2\}, 2 \leq j \leq n, \\
& C\left(u_{1}\right)=\{n, n+1, n+2, \cdots, 2 n-1\} \cup\{1,2\}, \\
& \bar{C}\left(u_{1}\right)=\{1,2, \cdots, n-1\} \backslash\{1,2\}, \\
& \bar{C}\left(u_{2}\right)=\{1,2, \cdots, n-1\} \backslash\{2,3\}, \\
& \quad \cdots \\
& \bar{C}\left(u_{i}\right)=\{1,2,3, \cdots, n-1\} \backslash\{i, i+1\}, \\
& \forall 2 \leq i \leq n-2, \\
& \bar{C}\left(u_{n-1}\right)=\{1,2,3, \cdots, n-1\} \backslash\{n-1,1\} .
\end{aligned}
$$

We can see that

$$
\begin{aligned}
& \bar{C}\left(u_{i}\right) \not \subset \bar{C}\left(v_{j}\right), \quad 1 \leq i \leq n-1, \quad 1 \leq j \leq n, \\
& \bar{C}\left(v_{j}\right) \not \subset \bar{C}\left(u_{i}\right), \quad 1 \leq i \leq n-1, \quad 1 \leq j \leq n, \\
& \bar{C}\left(u_{i}\right) \not \subset \bar{C}\left(u_{j}\right), \quad 1 \leq i \neq j \leq n-1,|\quad| i-j \mid=1, \\
& \bar{C}\left(u_{1}\right) \not \subset \bar{C}\left(u_{n-1}\right), \bar{C}\left(u_{n-1}\right) \not \subset \bar{C}\left(u_{1}\right), \\
& \bar{C}\left(v_{i}\right) \not \subset \bar{C}\left(v_{j}\right), \quad 1 \leq i \leq n, \quad 1 \leq j \leq n, \quad i \neq j .
\end{aligned}
$$

That is to say, all the vertices have color sets that are not contained in other color set of the adjacent vertices. So the given $f$ is a $(2 n-1)-S A$ for $C_{n-1} \vee K_{n}$.

So $\chi_{s a}^{\prime}\left(C_{n-1} \vee K_{n}\right)=2 n-1$.

Example 2 For $n=4$, then $\chi_{s a}^{\prime}\left(C_{3} \vee K_{4}\right)=7$.

In fact, we defined the mapping $f$ from $E\left(C_{3} \vee K_{4}\right)$ to $\{1,2,3,4,5,6,7\}$ as below:

$$
\begin{aligned}
& f\left(v_{1} v_{2}\right)=f\left(v_{3} v_{4}\right)=f\left(u_{1} u_{3}\right)=1, \\
& f\left(v_{1} v_{2}\right)=f\left(v_{2} v_{4}\right)=f\left(u_{1} u_{2}\right)=2, \\
& f\left(v_{1} v_{4}\right)=f\left(v_{2} v_{3}\right)=f\left(u_{2} u_{3}\right)=3, \\
& f\left(u_{1} v_{1}\right)=4, \quad f\left(u_{1} v_{2}\right)=5, \quad f\left(u_{1} v_{3}\right)=6, \quad f\left(u_{1} v_{4}\right)=7, \\
& f\left(u_{2} v_{1}\right)=5, \quad f\left(u_{2} v_{2}\right)=6, \\
& f\left(u_{2} v_{3}\right)=7, \quad f\left(u_{2} v_{4}\right)=4, \\
& f\left(u_{3} v_{1}\right)=6, \quad f\left(u_{3} v_{2}\right)=7, \\
& f\left(u_{3} v_{3}\right)=4, \quad f\left(u_{3} v_{4}\right)=5 .
\end{aligned}
$$

We can see that

$$
\begin{aligned}
& \bar{C}\left(v_{1}\right)=\{6,7\}, \bar{C}\left(v_{2}\right)=\{7,4\}, \\
& \bar{C}\left(v_{3}\right)=\{4,5\}, \bar{C}\left(v_{4}\right)=\{5,6\}, \\
& \bar{C}\left(u_{1}\right)=\{3\}, \bar{C}\left(u_{2}\right)=\{1\}, \bar{C}\left(u_{3}\right)=\{2\} .
\end{aligned}
$$

Obviously, $f$ is a 7-SA for $C_{3} \vee K_{4}$.

So $\chi_{s a}^{\prime}\left(C_{3} \vee K_{4}\right)=7$.

\section{Conclusion}

Coloring problem is a classical difficult problem of graph theory. Smarandachely adjacent- vertex-distinguishing proper edge coloring was first put forward by Zhang Zhong-fu in 2008. A lot of problems need to be solved urgently, such as finding out the smarandachely adjacent-vertex-distinguishing proper edge chromatic number, such as how smarandachely adjacent-vertex-distinguishing proper edge chromatic number 
changes when the vertices $\mathrm{n}$ grows.

In the paper, we deduce the smarandachely adjacent-vertex -distinguishing proper edge chromatic number of the joint graph $C_{m} \vee K_{n}$ by the methods of combination analysis and reduction to absurdity, also the method of apagoge.

\section{References}

[1] Liu Shun-qin, Chen Xiang-en. "Smarandachely Adjacent-Vertex-distinguishing proper edge coloring of $\bar{K}_{m} \vee K_{n}$ ". vol.41, pp. 155-158. August. 2015.

[2] Bondy. J .A. MURTY U S R. Graph Theory. London. Springer.2008.

[3] Zhang Zhong-fu, Chen Xiang-en, Li Jing-wen. "On adjacent -vertex-distinguishing total coloring of graphs." Sci. China. Ser. vol. 48. pp. 289-299. June. 1997.

[4] Chen Xiang-en, Zhan-fu, "Adjacent-Vertex-Distinguishing Total Chromatic Number of $P_{m} \times K_{n}$," Journal of Mathematical Research and Exposition, Dalian. vol. A26, pp. 489-494. August. 2015.

[5] Liu Shun-qin, Chen Xiang-en. "Smarandachely Adjacent-Vertex-distinguishing proper edge coloring of $\bar{K}_{3} \vee K_{n}{ }^{\prime}$. vol.37,pp.139-145.April.2011.

[6] Tian Jing-jing. "The Smarandachely Adjacent -Vertex -Eege Coloring of Some Mycielski's Graph". J. of. Math (PRC). vol. 32. pp. 723-728. April 2012.

[7] Qiang Hui-ying, Li mu-chun. "A Bound on Vertex Distinguishing Total Coloring of Graphs with Distance Constrant for Recurrent Event Data".Acta Mathematicae Applicatae Sinica. vol. 34. pp. 554-559. May. 2011.

[8] Tian Jing-jing, Deng Fang-an. “Adjacent Vertex-Distinguishing VE-Total Chromatic Number of the Crown Graph $C_{m} \cdot F_{n}$ and $C_{m} \cdot C_{n}$ ". Mathematics in Practice and Theory. vol. 41. pp. 189-192. August. 2011.
[9] Tian Jing-jing. "The Smarandachely Adjacent -Vertex -Eege Coloring of Some Mycielski's Graph". J. of. Math(PRC). vol. 32. pp. 723-728. April 2012.

[10] Lin Sun, Xiaohan Cheng, Jianliang Wu. "The Adjacent Vertex Distinguishing Total Coloring of Planar Graphs without Adjacent 4-cycles" J. Comb. Optim. DOI 10.1007/S10878-016-0004-1.11 March.2016.

[11] Xiaohan Cheng, Guanghui Wang, Jianliang Wu. "The Adjacent Vertex Distinguishing Total Chromatic Numbers of Planar Graphs with $\Delta=10$ ” J. Comb. Optim. DOI. 1007/S10878-016-9995-x.04 February.2016.

[12] Huijuan Wang, Bin Liu, Yan Gu, Xin Zhang."Total Coloring of Planar Graphs without Adjacent short cycles" J. Comb. Optim. DOI.1007/S10878-015-9954-y.16. September. 2015.

[13] Liu Hua, Feng Jianhua, Ma Shaoxian.”Adjacent Vertex-Distinguishing Edges coloring of $S_{m} . S_{n}$ " Journal of East China Jiao-tong University. vol. 24. pp. 157-158. October. 2007.

[14] Zhang Donghan, Zhang Zhongfu. "The Upper Bound of Adjacent Vertex Strongly Distinguishing Total Chromatic Number of the Graph". Advances of Mathematics. vol. 40. No. 2. pp. 168-172. April. 2011.

[15] Zhang Z F, Liu L Z, Wang J F. “Adjacent strong edge coloring of graphs”. Appl Math Lett. vol. 15. pp. 623-626. April. 2002.

[16] Shunqin Liu. "Several Kinds of Chromatic Numbers of Multi-fan Graphs". Applied and Computational Mathematics. vol. 5. No. 3. pp. 133-137. June. 2016.

[17] Wang YQ, Sun Q, Tao X, Shen L.'Plane graphs with maximum degree 7 and without 5-cycles with chords are 8-totally-colorable. Sci. China A. vol. 41. pp. 95-104. February. 2011.

[18] Wang B, Wu JL, Wang HJ. "Total coloring of planar graphs without chordal short cycles". Graphs Comb. Optim. vol. 60: 777-791. 2014. 